\title{
Neuronal-glial plasticity in gonadotropin-releasing hormone release in adult female rats: role of the polysialylated form of the neural cell adhesion molecule
}

\author{
Jyoti Parkash and Gurcharan Kaur \\ Neurochemistry and Neuroendocrinology Laboratory, Department of Biotechnology, Guru Nanak Dev University, Amritsar-143005, Punjab, India \\ (Requests for offprints should be addressed to G Kaur; Email: gurcharank@yahoo.com)
}

\begin{abstract}
The gonadotropin-releasing hormone $(\mathrm{GnRH})$ neurosecretory system undergoes marked structural and functional changes during the ovarian cycle. The aim of this study was to examine the neuroanatomical relationship between GnRH neurons and a polysialylated form of neural cell adhesion molecule (PSA-NCAM), a known marker of neuronal plasticity. Using immunohistofluorescent dual labeling, we determined that axon terminals of $\mathrm{GnRH}$ in the median arcuate nucleus (ME-ARC) region of the hypothalamus in the proestrous phase of the estrous cycle were intimately associated with PSA-NCAM. To further examine whether PSA-NCAM expression associated with GnRH neuron terminals varies in conjugation with cyclic changes in ovarian steroid hormone levels, we examined GnRH and PSA-NCAM dual expression in ovariectomized (OVX) and estrogen-progesterone-primed OVX (EBP-OVX) rats. The expression of PSA-NCAM immunoreactivity associated with the GnRH neurons in the
\end{abstract}

proestrous phase and EBP-OVX rats was significantly higher than during the diestrous phase and in OVX rats where GnRH secretion declines. We further examined whether the structural changes in $\mathrm{GnRH}$ axon terminals in the ME-ARC region are also associated with glial plasticity. By extension and retraction of the glial processes, the GnRH neuron terminals in the ME-ARC region could undergo dynamic plastic changes that control $\mathrm{GnRH}$ release during the proestrous phase. PSA-NCAM expression was also seen on glial cells in the ME-ARC region. The close association between PSA-NCAM on GnRH and glial cells in the ME-ARC region of the hypothalamus in the rat showed dynamic structural changes in GnRH neuron terminals during the estrous cycle. These observations suggested that PSA-NCAM may act as a molecular substrate to promote neuroplastic changes in the GnRH neurosecretory system.

Journal of Endocrinology (2005) 186, 397-409

\section{Introduction}

The gonadotropin-releasing hormone $(\mathrm{GnRH})$ system in the brain constitutes the final common pathway for the central regulation of pituitary gonadotropin release (Sarkar et al. 1976). GnRH is synthesized in neuronal cell bodies distributed diffusely in the hypothalamic preoptic area and is secreted from the external zone of the median eminence-arcuate (ME-ARC) region by an increase in the length of the axons (King et al. 1995, Kaur et al. 2002). In females, the preovulatory surge of luteinizing hormone $(\mathrm{LH})$ is initiated by an abrupt increase in GnRH release, which is preceded by GnRH axon terminal contact with the perivascular space directly (Prevot et al. 1999). A rise in circulating estradiol precedes the preovulatory LH surge and the ovarian cycle is also accompanied by a fall in the number of axosomatic synapses on randomly selected arcuate nucleus neurons by the morning of estrus, with a return to the usual levels by the morning of the next day (Naftolin et al. 1996). Studies of morphological and physiological plasticity in the hypothalamus are changing our earlier concepts about the structure-function relationship in the adult mammalian brain. It has recently become clear that fluctuating physiological conditions have the power to reversibly alter the structural relationships among the neurons and glial cells in the hypothalamus, as well as the functional pathways over which information is transmitted to the neuroendocrine neurons.

The GnRH-secreting neurons in mammals project principally to the median eminence (ME) where they abut in the pericapillary space of the primary hypophyseal portal plexus. Prevot et al. (1999) demonstrated for the first time the existence of cellular conformational changes in $\mathrm{GnRH}$ neuron terminals and the cells of the basal lamina in the external zone of the ME, and their quantified data revealed a statistically significant increase and the presence of contacts between GnRH neuroendocrine terminals and the parenchymatous basal lamina in the proestrous phase and an absence of these contacts in the diestrous phase. The dynamic plasticity changes, which occur in the 
external zone of the ME-ARC region on the day of proestrus, could be induced directly by gonadal steroids, since there is a dramatic increase in plasma estrogen levels from diestrus to the afternoon of proestrus prior to $\mathrm{LH}$ surge release.

The hypothalamus arcuate nucleus expresses high levels of polysialic acid (PSA) characterizing a highly sialylated isoform of the neural cell adhesion molecule (PSANCAM) and maintains a high capacity for neuroplastic changes in the adult brain. The large quantities of PSANCAM greatly reduce cell adhesion and render this particular cell surface molecule a key candidate to intervene in dynamic neuronal phenomena, such as synaptic plasticity. The modulation of adhesion arises from the length of the PSA, as this molecule has been shown to decrease NCAM homophillic binding and thereby attenuate cell adhesion. It has been proposed that this negative modulatory role of PSA helps the cells to detach from their neighbors, thus allowing them to respond to guidance or targeting cues, or to exhibit the plastic interactions required for motility (for review see Rutishauser \& Landmesser 1996). PSA also appears to intervene in the activation of second messenger pathways and, therefore, in intracellular signaling (Doherty et al. 1991). During neuronal development, PSA-NCAM expression is closely correlated with dynamic cellular phenomena underlying neuronal migration, axon path finding, targeting and fasciculation (for reviews see Fryer \& Hockfield 1996, Rutishauser \& Landmesser 1996, Yoshida et al. 1999). In the adult brain, PSA-NCAM continues to be expressed in neurons and glia capable of functional and morphological plasticity (Theodosis et al. 1991, Bonfanti et al. 1992, Bonfanti \& Theodosis 1994). In addition, PSA-NCAM re-expression has been noted in both neurons and glia in response to lesioning (Olsen et al. 1995, Bonfanti et al. 1996).

It is now well accepted that astrocytes play a fundamental role in the modulation of neuronal activity and metabolism (Araque et al. 1998) and local changes in neuronal activity are linked to modification of the expression of glial fibrilary acidic protein (GFAP). The GnRH neurons are extensively associated with non-neuronal elements of specifically glial cells (Fernadaz-Galaz et al. 1999). GFAP is a component of the intermediary filaments and increase in its expression is associated with the growth of astrocytic processes that may result in structural remodeling in the central nervous system (CNS) (Kohama et al. 1995, Stone et al. 1998). Glial processes seem to provide a permissive substrate for GnRH axonal extension, and the presence of a chemotropic factor, i.e. basic fibroblast growth factor (bFGF), provides specificity in the ME, which underlies the accurate navigation of the growing axon (Silverman et al. 1991). The fact that the GnRH axon displays a remarkable degree of outgrowth during a time of extensive glial hypertrophy and hyperplasia suggests that glial cells may play a facilitatory or permissive role in $\mathrm{GnRH}$ axonal extension (Silverman et al. 1991). Glial cell have been shown to regulate $\mathrm{GnRH}$ secretion via two related mechanisms, plastic rearrangements and direct cell-cell communication and, both mechanisms require the participation of growth factors (Ojeda et al. 2003). The pubertal activation of pulsatile $\mathrm{GnRH}$ secretion is not initiated within the GnRH neuronal network itself, but instead is brought about by the molecular and cellular events that take place in the neuronal and astro-glial network functionally connected to GnRH neurons. The present study was designed to explore the potential for PSA-NCAMmediated plasticity in the GnRH neuron in the adult rat, which shows cyclic changes in the structure and function of the GnRH system (Kaur et al. 2002). To further elucidate the role of astro-glial cells in the cyclic changes in GnRH neuron structure and function, we studied the expression of GnRH and GFAP in the proestrous and diestrous phase of the rat estrous cycle. As a first step, we co-localized GnRH and PSA-NCAM and GFAP and PSA-NCAM in the ME-ARC region of proestrous and diestrous phase rats by immunohistofluorescent dual staining. Using dual immunostaining of GnRH and GFAP, we further determined whether astro-glial cell processes associated with the GnRH neurons vary in conjunction with cyclic changes in GnRH neuronal terminals. To further test the role of steroid hormones in the cyclic neuronal plasticity of the $\mathrm{GnRH}$ neuron, we repeated these experiments in ovariectomized (OVX) and estrogen-progesterone-primed OVX (EBP-OVX) rats.

\section{Materials and Methods}

Wistar strain female albino rats of 3-5 months of age and weighing 200-250 g were procured from the National Institute of Nutrition, Hydrabad, India. Animals were housed three per cage in a temperature and humidity controlled environment on a $12 \mathrm{~h}$ light: $12 \mathrm{~h}$ darkness cycle with free access to food and water. The estrous cycle was monitored by daily inspection of vaginal cytology. After at least three to four complete 4-day cycles, the animals were killed between 1500 and $1600 \mathrm{~h}$ on diestrous $(n=12)$ and proestrous $(n=12)$ days. Another group of rats was ovariectomized after surgically removing both the ovaries under aseptic conditions and also a sham control group in which only lateral cuts were given and ovaries were not removed in adult cycling female rats of same age group was defined. Experiments were carried out 4-6 weeks after ovariectomy. Half of the rats were given $7.5 \mathrm{mg}$ estradiol benzoate (EB) (Sigma) subcutaneously in corn oil at $1000 \mathrm{~h}$. Progesterone $(5 \mathrm{mg}$ ) (Sigma) was administered subcutaneously in corn oil $48 \mathrm{~h}$ after the EB treatment (EBP-treated rats). All studies were performed between 1330 and $1430 \mathrm{~h}$ on the progesterone treatment day when the steroid-induced LH surge release occurs (Berglund et al. 1988). Animal care and procedures were followed in 
accordance with the guidelines of the Institutional Animal Ethical Committee of Guru Dev University.

\section{Procurement of antibodies for immunofluorescence}

The primary antibodies used for the immunohistofluorescence analysis were monoclonal anti-GFAP (clone CA-5; Sigma) and monoclonal antibody specific for PSA-NCAM which was a generous gift from Dr Tatsunori Seki (Juntendo University School of Medicine, Japan).

For immunohistofluorescent localization of $\mathrm{GnRH}$, LR1, an anti-GnRH antibody (a generous gift from G Tramu, CNRS, University of Bordeaux, Bordeaux, France) was used. The secondary antibodies used were Texas Red-conjugated (TRITC) goat anti-rabbit IgG at a dilution of 1:200 for GnRH, fluorescein isothicyanateconjugated (FITC) goat anti-mouse IgG at a dilution of 1:200 for GFAP and goat anti-mouse (FITC) $\operatorname{IgM} \mu$-chain specific at a dilution of 1:128 from Sigma for PSA.

\section{RIAs for LH and follicle-stimulating hormone (FSH) levels}

Serum LH and FSH levels were determined in duplicate using a double-antibody RIA technique. Blood samples from the trunk were clotted at $4{ }^{\circ} \mathrm{C}$, centrifuged for $10 \mathrm{~min}$ at 2500 r.p.m., and the sera separated and stored frozen until hormone assay. Serum LH and FSH levels were determined by the RIA method of Rao \& Mahesh (1986). EBP-OVX rats showing a serum LH level of $8.5 \mathrm{ng} / \mathrm{ml}$ and an FSH level of $10.7 \mathrm{ng} / \mathrm{ml}$ were selected for immunohistofluorescent experiments. OVX rats showing a serum LH level of $1.7 \mathrm{ng} / \mathrm{ml}$ and an FSH level of $3.5 \mathrm{ng} / \mathrm{ml}$ were further considered for experimentation.

\section{Immunofluorescent staining}

For immunofluorescent staining of GnRH+PSA-NCAM, GnRH+GFAP and GFAP+PSA-NCAM in the MEARC region, brains were perfused transcardially with $4 \%$ paraformaldehyde in phosphate buffer $(0 \cdot 1 \mathrm{M})$. Brains were kept in the fixative solution overnight at $4{ }^{\circ} \mathrm{C}$ and then cryopreserved in $20 \%$ and $30 \%$ sucrose in phosphate buffer each for $24 \mathrm{~h}$ at $4{ }^{\circ} \mathrm{C}$. Coronal sections (20 $\mu \mathrm{m}$ thick) were cut using a cryostat microtome and sections were mounted on gelatin-coated glass slides and treated in the following manner: three $15-\mathrm{min}$ washes in $0 \cdot 1 \mathrm{M}$ phosphate-buffered saline (PBS), $\mathrm{pH} 7 \cdot 4$ and $30 \mathrm{~min}$ in $0.3 \%$ Triton $\mathrm{X}-100$ in $0.1 \mathrm{M}$ PBS (PBST) for permeabilization. The sections were then washed with $0 \cdot 1 \%$ PBST for $15 \mathrm{~min}$. After washing, sections were preincubated for $1 \mathrm{~h}$ at room temperature in a blocking solution (5\% normal goat serum in PBS with $0.3 \%$ Triton X-100 and $0 \cdot 01 \%$ sodium azide). The sections were incubated with a mixture of primary antibodies, GnRH+PSA-NCAM, $\mathrm{GnRH}+\mathrm{GFAP}$ or GFAP+PSA-NCAM with appropriate dilutions of anti-GnRH (1:10 000), anti-PSA-NCAM
(1:500), anti-GFAP (1:500) in $0 \cdot 1 \%$ Triton $\mathrm{X}-100$ and $1 \%$ bovine serum albumin-PBS for $60 \mathrm{~h}$ at $4{ }^{\circ} \mathrm{C}$. Specificity of staining for GnRH, PSA and GFAP was determined by negative staining control procedures without adding primary antibodies to the incubation buffer. Mouse monoclonal PSA-NCAM antibody 12E3 (IgM) was a gift of a sample raised in the laboratory of Dr T Seki. The antibody was characterized by Seki and Arai using anti-PSA antibodies as well as by neuraamnidas (endo-N) treatment (Seki \& Arai 1991); the antibodies have strict specificity for PSA residues. The sections were then washed for $15 \mathrm{~min}$ with four changes of $0 \cdot 1 \%$ PBST at room temperature. The sections were incubated with specified secondary antibodies: anti-rabbit IgG for GnRH (TRITC with a dilution of 1:200), anti-mouse IgG for GFAP (FITC with a dilution of 1:200), anti-mouse IgM (FITC with a dilution of 1:128) for PSA-NCAM and anti-mouse $\operatorname{IgG}$ for GFAP (TRITC with a dilution of 1:200) in $0 \cdot 32 \%$ Triton-PBS for $2 \mathrm{~h}$. Sections were then washed with 0.01\% PBST for $15 \mathrm{~min}$. Tissue sections were then cover slipped using the appropriate anti-fading mounting medium (500 $\mathrm{mg}$ propyl-gallate, $20 \mathrm{ml}$ ethanol/PBS ( $\mathrm{pH} 7 \cdot 4$ ) (1:1) and $90 \mathrm{ml}$ glycerol) for fluorescence detection.

\section{Data analysis}

The dual-stained sections were examined using a Nikon fluorescent microscope with dual as well as single band filters. Images were captured using a Cool Snap CCD camera (Media Cybernetics, Silver Spring, MD, USA) and the pictures were analyzed using Image Pro-Plus software version 4.5.1 from Media Cybernetics. The extent of PSA-NCAM immunoreactivity associated with GnRH and GFAP immunoreactivity was quantified using two separate methods. The first analysis determined the overall density of GnRH, PSA-NCAM and GFAP immunoreactivity in 58 randomly selected fields in the ME-ARC region in each section using the count/size command of the Image Pro-Plus software. Five consecutive sections each from four to five animals in all groups were used for data analysis. An area of interest was selected at five to eight locations within the ME-ARC region on each section and density measurements were made for dual as well as for single staining of GnRH+PSA-NCAM, GnRH+GFAP or GFAP+PSA-NCAM.

The second method of analysis estimated the colocalization of GnRH and PSA-NCAM as well as of GFAP and PSA-NCAM using the co-localization command of the Image Pro-Plus software. This command measures the co-localization coefficient of two or more molecules (tagged with different fluorescent dyes) in precisely the same space. The percent difference in co-localization of GnRH+PSA-NCAM or GFAP+PSANCAM in proestrous and OVX-EBP rats as compared with the diestrous and OVX rats respectively was calculated and are presented as histograms. 

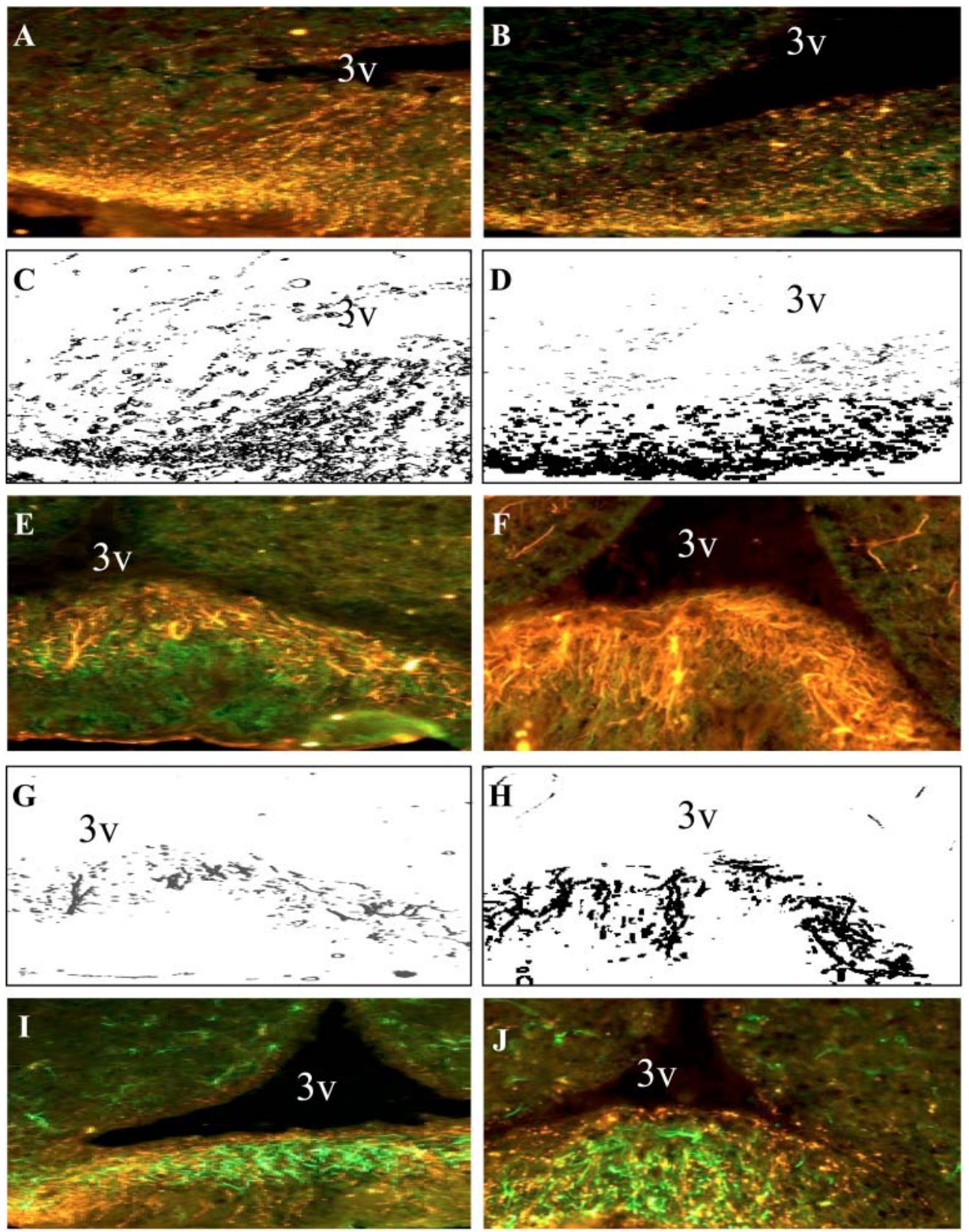


\section{Statistical analysis}

The values are expressed as means \pm S.E.M. The data were analysed using Student's $t$-test to determine the statistical significance. In the present study, different brain markers were studied in proestrous and diestrous rats in one group and OVX-EBP, OVX and sham controls in the second group. The statistically significant change in OVX-EBP and OVX rats in comparison with the sham control rats is shown in the figures by $\mathrm{a}, \mathrm{b}, \mathrm{c}$ or $\mathrm{d}$. The statistically significant changes in OVX-EBP in comparison with OVX rats is also shown in the figures by a', c' or d'. For each parameter, the mean \pm S.E.M. was determined in each group. Differences were considered statistically significant if $P<0 \cdot 05$.

\section{Results}

All data reported in the present study are restricted to adult female rat forebrain $\mathrm{ME}-\mathrm{ARC}$ regions of the hypothalamus. Using double immunostaining microscopy, coexpression of PSA-NCAM with GnRH or GFAP and co-distribution of GnRH and GFAP were examined in the diestrous and proestrous phases of cycling rats as well as in the sham control, OVX and EBP-OVX rats. Brain sections incubated in the absence of primary antibodies mixture showed no immunostaining.

\section{$G n R H$ immunoreactivity in the $M E-A R C$ region of the hypothalamus}

The immunofluorescent staining for GnRH using antiGnRH LR1 antibody showed a marked increase in the length and branching of GnRH axon terminals in the ME-ARC region in the proestrous phase as well as in EBP-OVX and sham control rats. On qualitative analysis, many GnRH terminals were seen to emit very thin processes in the direction of the perivascular space in the proestrous and EBP-OVX rats. GnRH terminals in the $\mathrm{ME}-\mathrm{ARC}$ region were heavily stained in the proestrous phase and EBP-OVX rats, indicating that these are the sites of active $\mathrm{GnRH}$ secretion. In the $\mathrm{ME}-\mathrm{ARC}$ region from proestrous and EBP-OVX rats, GnRH nerve endings were also seen to make close contact with the parenchymatous space. Results are presented in Figs 1A and $3 \mathrm{~A}$ and $\mathrm{C}$ (proestrous, EBP-OVX and sham control respectively) and Figs $1 \mathrm{~B}$ and $3 \mathrm{~B}$ (diestrous and OVX respectively).

\section{Co-localization of $\mathrm{GnRH}+\mathrm{PSA}-\mathrm{NCAM}$ expression in proestrous and diestrous rats}

In the ME-ARC region from proestrous rats, dense PSA-NCAM immunoreactivity was identified along the lateral border of the external zone of the ME, which was co-localized with a region of intense $\mathrm{GnRH}$ immunoreactive axon terminals. Double staining for $\mathrm{GnRH}$ and PSA-NCAM in this phase revealed that heavy PSANCAM expression was co-localized with GnRH immunoreactivity as is evident from the yellow staining appearing in co-localized areas. Results are presented in Fig. 1A. On the other hand, GnRH and PSA-NCAM expression was much lower in diestrous phase rats (Fig. 1B). Results of co-localization analysis shown in Fig. 1C and D further illustrate that GnRH and PSA-NCAM were being expressed together. Using quantitative immunofluorescence analysis of staining intensity measurements, we showed a statistically significant increase $(P<0 \cdot 001)$ in the PSANCAM and GnRH dual staining in the ME-ARC region from the proestrous rats as compared with the diestrous phase rats (Fig. 2A). Similarly, an analysis of single staining for GnRH and PSA-NCAM showed significantly higher expression in proestrous as compared with diestrous phase rats (Fig. 2A). Comparison of co-localization of $\mathrm{GnRH}$ and PSA-NCAM in the ME-ARC region showed a

\footnotetext{
Figure 1 Photomicrographs of $20 \mu \mathrm{m}$ thick coronal section through the ME-ARC region of the hypothalamus; double immunostaining of the section for GnRH and PSA-NCAM is shown for the (A) proestrous and (B) diestrous phases. The intense PSA-NCAM and GnRH dual immunostaining was located along the external zone of the lateral portion of the ME in sections from proestrous as compared with diestrous phase rats. Note that the area where PSA-NCAM and GnRH immunoreactivities overlap appears as heavy (yellow) staining as shown in (A) and (B). Both immunoreactivities appear to be co-localized in the ventral arcuate nucleus and in the internal and external zone of the ME. Co-localization of GnRH and PSA-NCAM expression was analyzed using the co-localization function of Image Pro-Plus software and results of co-localization are shown in (C) proestrous and (D) diestrous stages. Photomicrographs of $20 \mu \mathrm{m}$ thick coronal section through the ME-ARC region of the hypothalamus; double immunostaining of the section for GFAP and PSA-NCAM is shown for (E) proestrous and (F) diestrous phases. The intense PSA-NCAM immunostaining was localized along the external zone of the ME in sections from proestrous phase rats whereas PSA-NCAM-ir was much lower in diestrous rats. The expression of GFAP immunostaining was localized along the external and internal zone of the ME in sections from (F) diestrous rats but in (E) proestrous phase rats GFAP expression was restricted to internal zone of the ME. Note that the area where PSA-NCAM and GFAP immunoreactivities overlap appears yellow as shown in (E) and (F). Co-localization of GFAP and PSA-NCAM expression was analyzed using the co-localization function of Image Pro-Plus software and results of co-localization are shown in $(G)$ proestrous and $(H)$ diestrous rats. Photomicrographs of $20 \mu m$ thick coronal section through the median eminence arcuate region; double immunostaining of the section for GnRH and GFAP is shown for (I) proestrous and $(\mathrm{J})$ diestrous phase rats. Immunostaining in both appeared to be co-distributed in the same area of ventral arcuate nucleus and of the internal and external zone of the ME. The expression of GFAP was observed in both the external and internal zone of the ME in diestrous rats but its expression was restricted to the internal zone of the ME in the proestrous rats. The expression of GnRH increased in proestrous as compared with diestrous phase rats. $3 \mathrm{v}$, third ventricle. Magnification $\times 50$. This figure appears in colour at http://joe.endocrinology-journals.org/content/vol186/issue2/.
} 

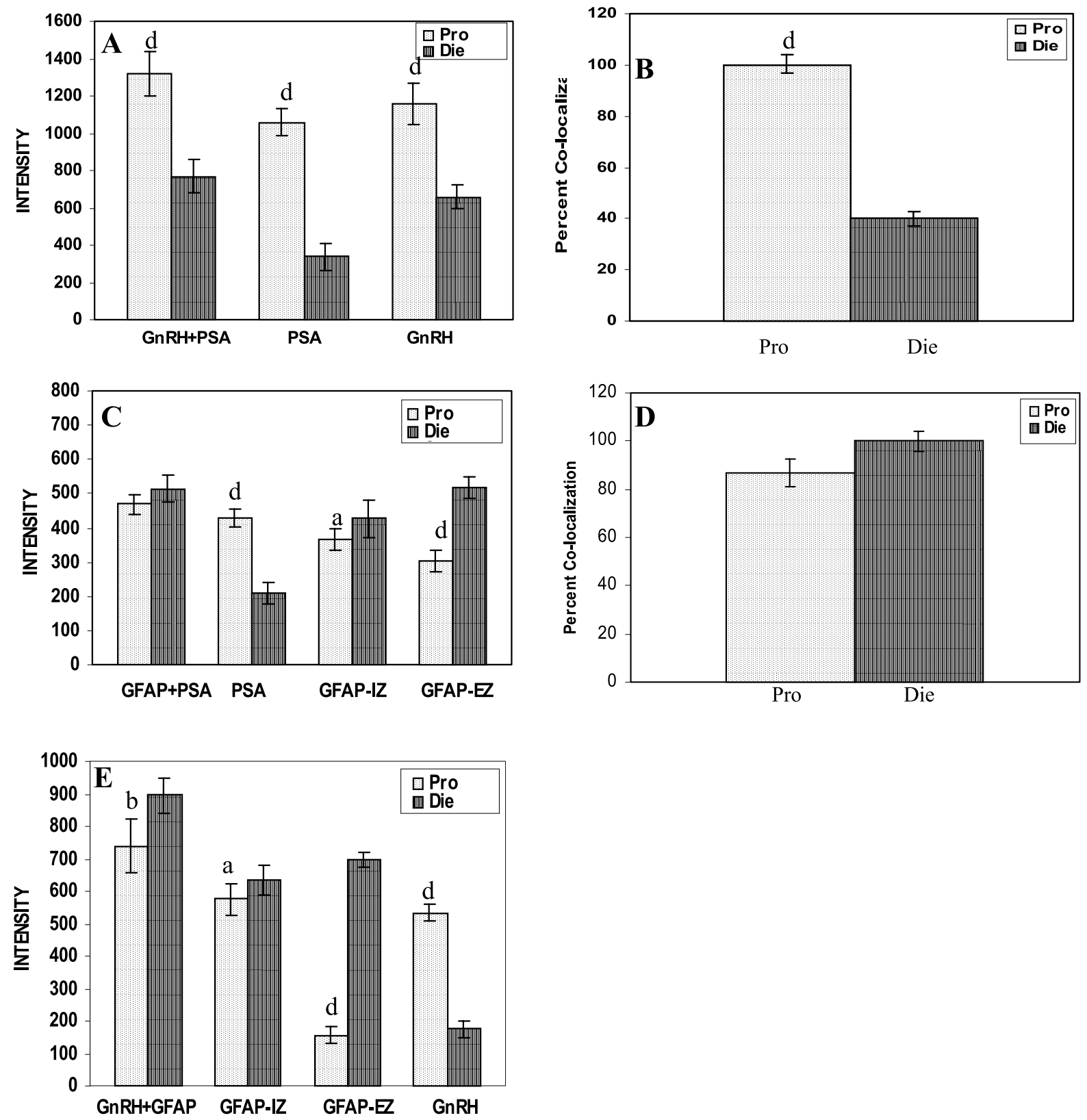

Figure 2 Intensity measurement data of the PSA-NCAM immunoreactivity associated with the GnRH axon terminals in the ME-ARC region of proestrous (dotted bars) and diestrous (lined bars) phase rats; (A) depicts the staining intensity of GnRH and PSA-NCAM in proestrous and diestrous rats. GnRH+PSA depicts the intensity data of dual-stained sections, PSA depicts PSA-NCAM immunoreactivity and GnRH depicts the GnRH immunoreactivity in proestrous and diestrous rats. (B) Quantitative data of percent change in co-localization of GnRH and PSA-NCAM in proestrous and diestrous rats was analyzed from Fig. 1C and D using the co-localization function of Image Pro-Plus software. Intensity measurement data of the PSA-NCAM immunoreactivity associated with the glial cell in the ME-ARC region of proestrous and diestrous phase rats; (C) depicts the staining intensity of GFAP and PSA-NCAM in proestrous and diestrous rats. GFAP+PSA depicts the intensity data of dual-stained sections, PSA depicts PSA-NCAM immunoreactivity and GFAP depicts the GFAP immunoreactivity in proestrous and diestrous rats in the IZ and EZ. (D) Quantitative data of percent change in co-localization of GFAP and PSA-NCAM in proestrous and diestrous rats was analyzed from Fig. $1 \mathrm{G}$ and $\mathrm{H}$ using the co-localization function of Image Pro-Plus software. Intensity measurement data of the GFAP immunoreactivity associated with the GnRH axon terminals in the ME-ARC region of proestrous and diestrous phase rats; (E) depicts the staining intensity of GnRH and GFAP in proestrous and diestrous rats. GnRH+GFAP depicts intensity data of dual-stained sections, GFAP depicts GFAP-ir in the IZ and the EZ and GnRH depicts the GnRH-ir in proestrous and diestrous rats. For each type of data analysis in (A), (C) and (E) measurements were performed on five to eight sections each from four to five proestrous and diestrous phase rats. All statistical analyses were performed using Student's t-test. Values are means \pm S.E.M.; $\mathrm{a}=P<0 \cdot 05, \mathrm{~b}=P<0 \cdot 02, \mathrm{~d}=P<0 \cdot 001 . \mathrm{Z}$, internal and $\mathrm{EZ}$, external zones of the ME. 
highly significant increase in percent co-localization in the proestrous phase rats as compared with the diestrous group (Fig. 2B).

\section{Co-localization of GFAP+PSA-NCAM expression in proestrous and diestrous rats}

In the ME-ARC region, double immunofluorescence staining in the proestrous phase of the estrous cycle showed that GFAP protein expression was restricted only to the internal zone of the ME-ARC but, in the case of diestrous rats, GFAP expression was observed in both the external and internal zone of the ME-ARC. PSA-NCAM immunoreactivity was identified along the internal and external zone of the ME (with more pronounced expression in the external zone) in proestrous rats whereas immunoreactive PSA-NCAM (PSA-NCAM-ir) was much lower in diestrous rats. Results are shown in Fig. 1E for proestrous rats and Fig. 1F for diestrous phase rats. Quantitative immunofluorescence analysis of staining intensity measurements showed an increase in the dual PSA-NCAM and GFAP staining in the ME-ARC region in the diestrous rats as compared with the proestrous phase rats. But an analysis of single staining for PSA-NCAM showed significantly higher expression in proestrous as compared with diestrous phase rats, whereas GFAP expression was much higher in diestrous as compared with proestrous rats. Results are presented in Fig. 2C. Comparison of co-localization of dual GFAP and PSA-NCAM in the internal zone of the ME-ARC region showed an insignificant change in percent co-localization in the diestrous and proestrous rats (Fig. 2D).

$\mathrm{GnRH}$ and GFAP expression in proestrous and diestrous rats

Immunofluorescence data indicated that $\mathrm{GnRH}$ expression increased significantly in the ME-ARC region of the hypothalamus in the proestrous phase as compared with the diestrous rats. GnRH axons were co-distributed with the glial elements in the internal zone of the ME in the proestrous phase, whereas GFAP staining was observed in both internal and external zones of the ME-ARC in diestrous phase rats, thus indicating reduced glial apposition with GnRH axon terminals in the parenchymatous space to facilitate $\mathrm{GnRH}$ release in the proestrous phase. The results of GnRH and GFAP dual immunostaining are presented in Fig. 1I for proestrous and Fig. 1J for diestrous rats. Using quantitative immunofluorescence analysis of staining intensity measurements, we showed a statistically significant increase in the GFAP staining in the ME-ARC region in the diestrous rats as compared with the proestrous phase rats. Results are presented in Fig. 2E. Similarly, an analysis of single staining for GnRH showed higher expression in proestrous as compared with diestrous phase rats but the reverse was observed for GFAP expression (Fig. 2E).
Co-localization of GnRH and PSA-NCAM expression in $O V X$, steroid-primed $O V X$ and sham control rats

In the ME-ARC region from EBP-OVX rats, dense PSA-NCAM immunoreactivity was identified along the lateral border of the external zone of ME, which was co-localized with a region of intense GnRH immunoreactive axons terminals. Double immunostaining for GnRH and PSA-NCAM in these rats revealed that heavy PSA-NCAM expression was co-localized with GnRH immunoreactivity as is evident from the yellow staining appearing in the co-localized area. Results are presented in Fig. 3A. On the other hand, GnRH and PSA-NCAM expression was much lower in OVX rats as well as in sham control rats (Fig. 3B and C). Results of co-localization analysis shown in Fig. 3D, E and $\mathrm{F}$ further illustrate that GnRH and PSA-NCAM were being expressed together. Results of co-localization analysis shown in Fig. 3D, E and F for OVX-EBP, OVX and sham control rats respectively further illustrate that GnRH and PSA-NCAM were being expressed in the same cell. Using quantitative immunofluorescence analysis of staining intensity measurements, we showed a statistically significant increase $(P<0 \cdot 001)$ in the PSA-NCAM and GnRH dual staining in the ME-ARC region from EBP-OVX rats as compared with OVX as well as with sham control rats (Fig. 4A). Similarly, an analysis of single staining for GnRH and PSA-NCAM showed significantly higher expression of these markers in EBP-OVX rats as compared with OVX and sham control rats (Fig. 4A). Comparison of co-localization of $\mathrm{GnRH}$ and PSA-NCAM in the ME-ARC region showed a highly significant increase in their percent co-localization in the EBP-OVX rats as compared with OVX and sham control rats (Fig. 4B).

Co-localization of GFAP and PSA-NCAM expression in OVX, steroid-primed OVX and sham control rats

In the ME-ARC region, double immunofluorescence staining in EBP-OVX rats during the estrous cycle showed that GFAP protein expression was restricted only to the internal zone of the ME-ARC but, in the case of OVX and sham control rats, GFAP expression was observed in both the external and internal zone of ME-ARC. PSANCAM immunoreactivity was identified along the internal and external zone of the ME (with more pronounced expression in the external zone) in EBP-OVX rats whereas PSA-NCAM-ir was much lower in OVX and sham control rats. The results of GFAP and PSA-NCAM immunostaining are presented in Fig. $3 \mathrm{G}$ for EBP-OVX and Fig. $3 \mathrm{H}$ for OVX and Fig. 3I for sham control rats. Results of co-localization analysis shown in Fig. 3J, K and L for OVX-EBP, OVX and sham control rats respectively further illustrate that GFAP and PSA-NCAM were being expressed in the same cell. Using quantitative immunofluorescence analysis of staining intensity measurements, 

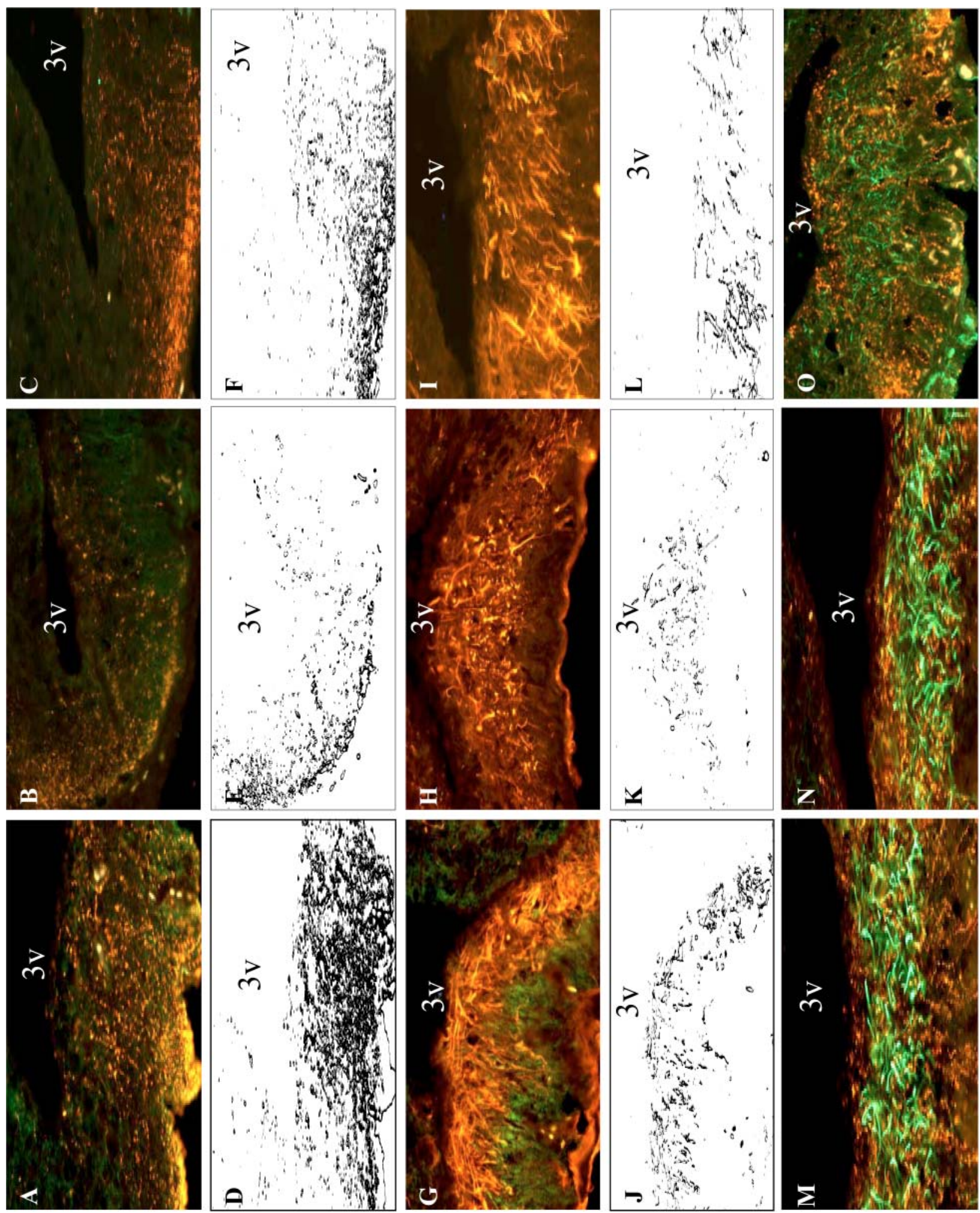
we showed a statistically insignificant increase in the dual PSA-NCAM and GFAP staining in the ME-ARC region in the OVX and sham control rats as compared with the EBP-OVX rats. Similarly, an analysis of single staining for PSA-NCAM showed a significantly higher expression in EBP-OVX as compared with OVX and sham control rats, whereas GFAP showed that expression was higher in the OVX and sham controls as compared with EBP-OVX rats. Results are presented in Fig. 4C. Comparison of co-localization of dual GFAP and PSA-NCAM in the internal zone of the ME-ARC region showed an insignificant change in percent co-localization in the OVX and EBP-OVX rats (Fig. 4D).

\section{GnRH and GFAP expression in OVX, steroid-primed $O V X$ and sham control rats}

Immunofluorescence data indicated that $\mathrm{GnRH}$ expression significantly increased in the ME-ARC region of the hypothalamus in the EBP-OVX as compared with the OVX and sham control rats. GnRH axons were codistributed with the glial elements in the internal zone of the ME in the EBP-OVX rats, whereas GFAP staining was observed in both internal and external zones of the ME-ARC in OVX and sham control rats, thus indicating reduced glial apposition with $\mathrm{GnRH}$ in the parenchymatous space to facilitate GnRH release in EBP-OVX rats. The results of GnRH and GFAP immunostaining are presented in Fig. $3 \mathrm{M}$ for EBP-OVX, Fig. 3N for OVX and Fig. $3 \mathrm{O}$ for sham control rats. Using quantitative immunofluorescence analysis of staining intensity measurements, we showed a significant increase in GFAP immunoreactivity in the internal as well as the external zone in the ME-ARC region in OVX and sham control rats, whereas GFAP immunoreactivity was restricted to the internal zone of EBP-OVX rats. Results are presented in Fig. 4E. Similarly, an analysis of single staining for
GnRH showed higher expression in EBP-OVX as compared with OVX rats but the reverse was observed for GFAP expression.

\section{Discussion}

In the present investigation we have demonstrated that the GnRH neuron terminals in the external zone of the ME undergo dramatic conformational changes resulting in the physical contact between GnRH nerve endings and parenchymatous basal lamina, so as to facilitate the entry of GnRH into the portal circulation during proestrus. Several previous studies using quantitative image analysis have shown dynamic transformation of individual GnRH terminals in the ME-ARC region of the hypothalamus as a function of gonadectomy as well as during different phases of the estrous cycle in rats (Prevot et al. 2000). The synaptic restructuring of the ME-ARC region during the proestrous phase of the estrous cycle indicates that the adult pattern of synaptic connections could be altered in the rat hypothalamus in relation to endocrine events, as is also evident from GnRH axon sprouting shown in Fig. 1A. Since neuroendocrine $\mathrm{GnRH}$ neurons are diffusely distributed throughout the hypothalamus, the ME-ARC region provides a common point of convergence for the control of GnRH neuronal activity and hence plasticity.

The present results have further shown that the $\mathrm{GnRH}$ neuron terminal-containing area, i.e. the ME-ARC region in the rat hypothalamus, retains the capacity to express PSA-NCAM in the adult brain, and also that PSA-NCAM expression shows cyclic changes in the estrous cycle of the rat. PSA-NCAM expression in the ME-ARC region during the proestrous phase may be responsible for the structural reorganization of the $\mathrm{GnRH}$ neuron terminals in the proestrous phase. Other compelling evidence has been reported in our earlier study

\footnotetext{
Figure 3 Photomicrographs of $20 \mu \mathrm{m}$ thick coronal section through the ME-ARC region of the thypothalamus; double immunostaining of the section for GnRH and PSA-NCAM is shown for (A) OVX-EBP, (B) OVX and (C) sham control rats. The intense PSA-NCAM and GnRH immunostaining was located along the external zones of the lateral portion of the ME in sections from OVX-EBP as compared with OVX and sham control rats. Note that the area where PSA-NCAM and GnRH immunoreactivities overlap appears as heavy (yellow) staining as shown in (A) and (B). Both immunoreactivities appear co-localized in the ventral arcuate nucleus and in the internal and external zone of the ME. Co-localization of GnRH and PSA-NCAM expression was analyzed using the co-localization function of Image Pro-Plus software and results of co-localization are shown in (D) OVX-EBP, (E) OVX and (F) sham control. Photomicrographs of 20 $\mu \mathrm{m}$ thick coronal section through the ME-ARC region of the hypothalamus; double immunostaining of the same section for GFAP and PSA-NCAM is shown for (G) OVX-EBP, (H) OVX and (I) sham control rats. The intense PSA-NCAM immunostaining was localized along the external zones of the ME in sections from OVX-EBP rats (A) whereas PSA-NCAM-ir was much lower in OVX sham control rats. The expression of GFAP immunostaining was localized along the external and internal zone of the ME in sections from (H) OVX rats and (I) sham control but in OVX-EBP rats GFAP expression was restricted to the internal zone of the ME as shown in $(\mathrm{G})$. Note that the area where PSA-NCAM and GFAP immunoreactivities overlap appears yellow as shown in $(\mathrm{G}),(\mathrm{H})$ and $(\mathrm{I})$. Co-localization of GFAP and PSA-NCAM expression was analyzed using the co-localization function of Image Pro-Plus software and results of co-localization are shown in (J) OVX-EBP, (K) OVX and (L) sham control. Photomicrographs of $20 \mu \mathrm{m}$ thick coronal section through the median eminence arcuate region; double immunostaining of the section for GnRH and GFAP is shown for (M) OVX-EBP, (N) OVX and (O) sham control rats. Both immunostainings appeared co-distributed in the same area of ventral arcuate nucleus and of the internal and external zones of the ME. The expression of GFAP was observed both in the external and internal zone of ME in OVX and sham control rats but its expression was restricted to the internal zone of ME in the OVX-EBP rats. The expression of GnRH increased in OVX-EBP as compared with OVX and sham control rats. $3 \mathrm{v}$, third ventricle. Magnification $\times 50$. This figure appears in colour at http://joe.endocrinology-journals.org/content/vol186/issue2/.
} 

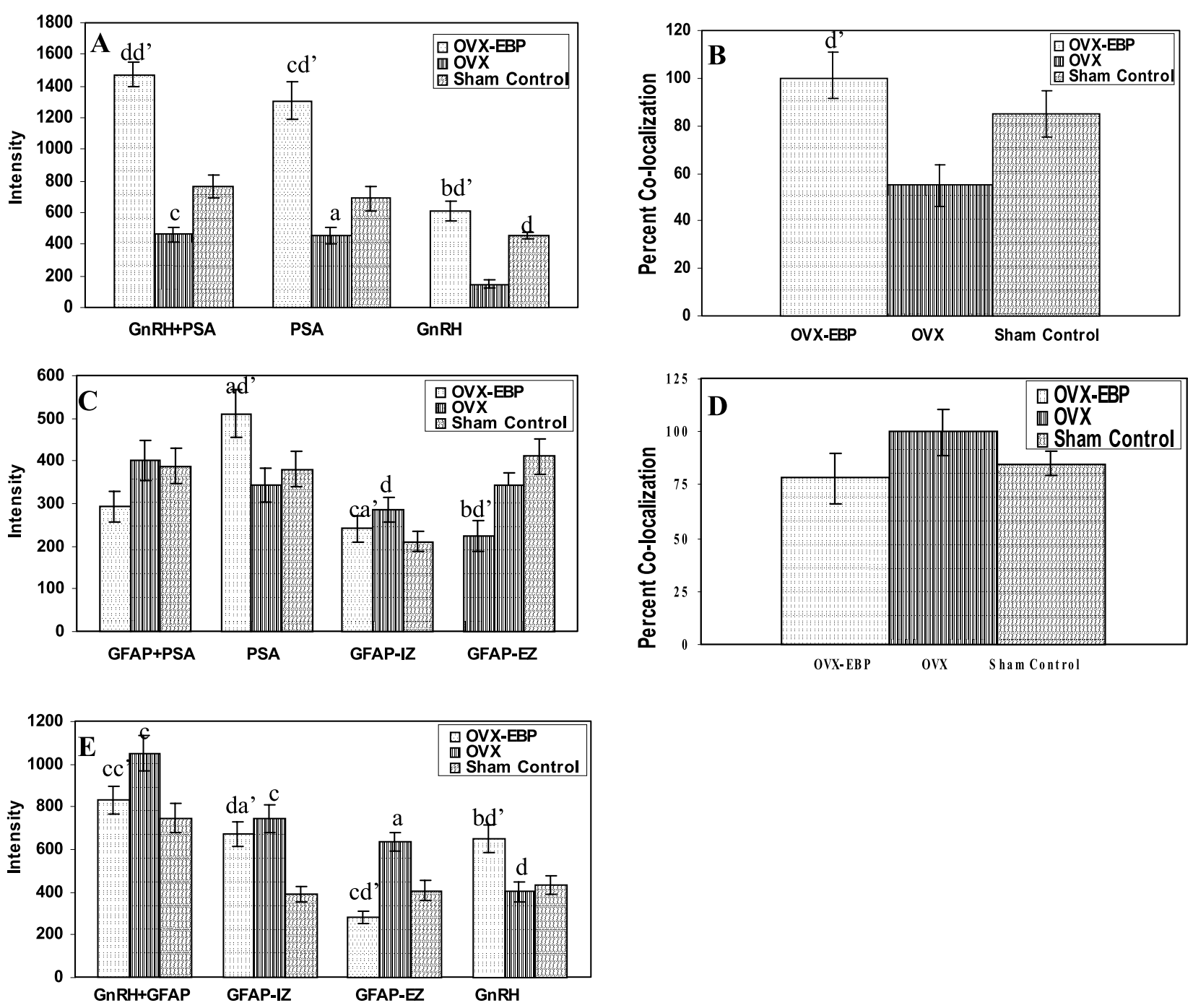

Figure 4 Intensity measurement data of the PSA-NCAM immunoreactivity associated with the GnRH axon terminals in the ME-ARC region of OVX-EBP (dotted bars) and OVX (lined bars); (A) depicts the staining intensity of GnRH and PSA-NCAM in OVX-EBP and OVX rats. GnRH+PSA depicts the intensity data of dual-stained sections, PSA depicts PSA-NCAM immunoreactivity and GnRH depicts the GnRH immunoreactivity in OVX-EBP and OVX rats. (B) Quantitative data of percent change in co-localization of GnRH and PSA-NCAM in OVX-EBP, OVX and sham control rats was analyzed from Fig. 3D, F and G using the co-localization function of Image Pro-Plus software. Intensity measurement data of the PSA-NCAM immunoreactivity associated with the glial cell in the ME-ARC region of OVX-EBP, OVX and sham control rats; $(C)$ depicts the staining intensity of GFAP and PSA-NCAM in OVX-EBP and OVX rats. GFAP+PSA-NCAM depicts intensity data of dual-stained sections, PSA depicts PSA-NCAM immunoreactivity and GFAP depicts the GFAP immunoreactivity in OVX-EBP, OVX and sham control rats. (D) Quantitative data of percent change in co-localization of GFAP and PSA-NCAM in OVX-EBP, OVX and sham control rats was analyzed from Fig. 3J, K and L using the co-localization function of Image Pro-Plus software. Intensity measurement data of the GFAP immunoreactivity associated with the GnRH axon terminals in the ME-ARC region of OVX-EBP and OVX rats; (E) depicts the staining intensity of GnRH and GFAP in OVX-EBP and OVX and sham control rats. GnRH+GFAP depicts intensity data of dual-stained sections, GFAP depicts GFAP co-distribution in the IZ and the EZ and GnRH depicts the GnRH immunoreactivity in OVX-EBP and OVX rats. For each type of data analysis in (A), (C) and (E), measurements were performed on five to eight sections each from four to five OVX-EBP, OVX and sham control rats. All statistical analyses were performed using Student's $t$-test. Values are means \pm S.E.M.; $\mathrm{a}, \mathrm{a}^{\prime}=P<0 \cdot 05, \mathrm{~b}=P<0 \cdot 02, \mathrm{c}, \mathrm{c}^{\prime}=P<0 \cdot 01, \mathrm{~d}, \mathrm{~d}^{\prime}=P<0 \cdot 001$. IZ, internal and EZ, external zones of the ME.

(Kaur et al. 2002) where we have shown that Western analysis of membrane extract from the ME-ARC region from proestrous phase rats revealed a PSAimmunoreactive band in the $150-250 \mathrm{kDa}$ range. The present immunohistofluorescent results have further indicated that PSA-NCAM expression within the hypothalamus is confined to the ME-ARC region. Viguie et al. (2001) reported that the amount of PSA-NCAM 
associated with GnRH neurons also increased in the breeding season of ewes. The preovulatory surge of $\mathrm{LH}$ is initiated by an abrupt increase in GnRH release (Levine 1997, Prevot et al. 2000). These observations are also supported by previous studies which have demonstrated the existence of dynamic plasticity changes in the external zone of the ME, which allows physical contacts between $\mathrm{GnRH}$ nerve endings and the perivascular space on the day of proestrus to facilitate the release of this neurohormone into pituitary portal blood (Alonso et al. 1997, Prevot et al. 1999, Prevot et al. 2000).

PSA-NCAM continues to be expressed in areas of the adult CNS, which shows the capacity for morphological and physiological plasticity (Seki \& Arai 1993). The ME-ARC region having GnRH neuron terminals seems to be a potential area of adult expression of PSA-NCAM. The molecular mechanism by which PSA intervenes to bring about dynamic changes in the GnRH neuron terminal remains unknown. In the GnRH neurosecretory system, steroid hormones seem to be an inductive factor to enhance PSA-NCAM expression and GnRH neuron terminals, as is evident from enhanced expression of PSA-NCAM in EBP-OVX rats as compared with OVX animals. We also observed greater expression of PSANCAM associated with GnRH terminals in the proestrous phase than during the diestrous phase. These observations clearly suggested that the expression of PSA-NCAM in the hypothalamic regions that regulate reproduction is more prevalent under a stimulatory period preceding $\mathrm{LH}$ surge release in the afternoon of proestrus or in EBP-OVX rats. Moreover, PSA-NCAM expression during the proestrous phase and in EBP-OVX rats was more pronounced in the external zone of the ME, i.e. towards the perivascular space (Figs 1A and 3A), which further indicates the permissive role of PSA-NCAM in GnRH release. Viguie et al. (2001) reported that, besides influencing synaptic rearrangements, PSA-NCAM might also promote rearrangement of cells in close vicinity to the $\mathrm{GnRH}$ neurons and their terminals. PSA-NCAM may serve to regulate the extracellular environment through the molecular interactions, which may facilitate or inhibit neuronal-glial remodeling.

Prevot et al. (1999) have reported that there is an increased contact of $\mathrm{GnRH}$ nerve terminals with the perivascular space in the ME region during proestrus, but it was not clear whether this increased contact was due to $\mathrm{GnRH}$ neurite extension or withdrawal of glial ensheathment. Another report (Garcia-Segura et al. 1988) has shown that estrogen induces a transient disconnection of axo-somatic inhibitory synapses in the arcuate nucleus during the estrous cycle. In the arcuate nucleus of female rats, changing circulating estrogen levels have been shown to reversibly reduce the number of axo-somatic GABA synapses, along with changing ensheathment of neuronal somata by astrocytes (Hoyk et al. 2001). These previous reports and our present results strongly suggest that glial cells provide an important tool for modulating the number of inputs onto GnRH neurons. Recent reports suggest that astrocytes have important neuroregulatory function in addition to their classic function of support and regeneration of neurons (Dhandapani et al. 2003). In addition to releasing neuroactive factors, astrocytes may also be capable of controlling GnRH neuronal function via regulation of hypothalamic synaptic plasticity. Glial ensheathment or extension and withdrawal of astrocytic processes may serve to modulate the synaptic contacts of $\mathrm{GnRH}$ neurons and their axon sprouting. The present result of dual staining of GnRH and GFAP in the ME-ARC region from cycling rats revealed reduced glial ensheathment of $\mathrm{GnRH}$ neuron terminals in the external zone of the ME region, whereas in diestrous phase animals GFAP staining was seen in both the external and internal zone of the ME (Fig. 1J). These observations suggested that withdrawal of glial ensheathment of GnRH neuron terminals in the external zone of the ME in the proestrous phase may facilitate the GnRH release process.

We have further shown that ovariectomy led to increased apposition of glial processes to the axon terminals of GnRH neurons in the ME-ARC region and a corresponding decrease of ensheathment in EBP-OVX rats. These observations suggested that alterations in the circulating gonadal steroid levels influence the morphology and function of glial cells. In a recent article, Galbiati et al. (2003) have reviewed the mechanism through which steroid hormone may influence the GnRH neurosecretory system. They have hypothesized that the two different types of signals regulating $\mathrm{GnRH}$-secreting neurons, i.e. steroid hormones and growth factors such as transforming growth factor (TGF)- $\beta$, TGF $\alpha$ and bFGF, might be integrated at the level of hypothalamic astrocytes. Several reports have indicated that glial cells influence the activity of hypothalamic GnRH-secreting neurons via the release of growth factors (Ma et al. 1994, Cavarretta et al. 1999, Galbiati et al. 2001). Estrogen has been shown to increase both the mRNA and protein levels of bFGF in hypothalamic astrocytes, and also the mRNA level of bFGF in the hypothalamus of OVX rats was elevated by estrogen treatment (Melcangi et al. 2001, 2002, Galbiati et al. 2002).

Additional evidence supporting a role for glia in modulating GnRH function comes from our present studies demonstrating the co-expression of GnRH with GFAP and of GFAP with PSA-NCAM, which varies during the estrous cycle. PSA-NCAM was expressed in both glia and neurons. Removal of PSA from the NCAM molecule in vivo in rats by using endo- $\mathrm{N}$ enzyme has been shown to block both the glial retraction and the increase in synaptic inputs on the magnocelluar neuroendocrine neurons during lactation and dehydration (Theodosis et al. 1999, Hoyk et al. 2001). An important indirect functional consequence of a modified glial coverage seems to permit the GnRH neuron terminals to undergo rearrangement. 
These neuronal and glial conformational changes in the ME-ARC region are reversible and occur in a cyclic fashion and they are accompanied by changing expression of PSA-NCAM expression on both GnRH neuron terminals and glial cells.

The present data clearly showed that the morphological changes observed in the GnRH neuron terminal in the ME-ARC region along with changes in their glial apposition certainly has physiological consequences. These alterations are a part of the GnRH neurosecretory mechanism, which is further supported by the fact that the ME-ARC region was strongly positive for PSA-NCAM in the proestrous phase as well as in OVX-EBP rats. PSA-NCAM expression was downregulated in the diestrous phase and in the OVX rats. These observations suggested that the neuronal-glial cell membrane interaction through a permissive factor, i.e. PSA-NCAM, is actively involved in the structural synaptic remodeling in adult animals. These processes are influenced by changes in gonadal steroid levels. The present results suggested a mechanism whereby large quantities of PSA on extracellular surfaces would attenuate adhesion via physical impedance or charge repulsion, thus allowing dynamic structural modifications in $\mathrm{GnRH}$ neurons. In several recent reports, PSA-NCAM has been considered a necessary permissive substrate which facilitates morphological neuronal and glial plasticity (Viguie et al. 2001, Jansen et al. 2003. Theodosis et al. 2004), allowing hypothalamic neurons and their synapses to undergo structural changes whenever the proper stimulus intervenes. Besides acting as a permissive molecular factor, PSA-NCAM, by permitting or inhibiting neuronal glial remodeling, may also be affecting the presence of astrocytic processes in the external zone of the ME. In conclusion, the GnRH neurosecretory system, by its physiological capacity to express PSA-NCAM, can undergo dynamic transformations of its axon terminals and their glial ensheathment. The GnRH system can serve as useful model to illustrate the molecular mechanisms of neuronal plasticity and its functional consequences in the adult brain.

\section{Acknowledgements}

We would like to thank Dr Tatsunori Seki for his generous supply of PSA-NCAM and Dr G Tramu for the antiGnRH antibody. This study was supported by a grant from the Department of Biotechnology, Government of India. The authors declare that there is no conflict of interest that would prejudice the impartiality of this scientific work.

\section{References}

Alonso G, Prieto M, Legrand A \& Chauvet N 1997 PSA-NCAM and B-50/ GAP-43 are co-expressed by specific neuronal system of the adult rat mediobasal hypothalamus that exhibit remarkable capacities for morphological plasticity. Journal of Comparative Neurology 384 181-199.

Araque A, Parpura V, Sanzgiri RP \& Hayden PG 1998 Glutamate dependent astrocyte modulation of synaptic transmission between cultured hippocampus neurons. European Journal of Neuroscience 10 2129-2142.

Berglund LA, Derendorf H \& Simpkins JW 1988 Desensitization of brain opiate receptor mechanisms by gonadal steroid treatments that stimulate luteinizing hormone secretion. Endocrinology 122 200-203.

Bonfanti L \& Theodosis DT 1994 Expression of polysialylated neural cell adhesion molecule by proliferating cells in the subependymal layer of the adult rat, in its rostral extension and in the olfactory bulb. Neuroscience 62 291-305.

Bonfanti L, Olive S, Poulain DA \& Theodosis DT 1992 Mapping of the distribution of polysialylated neural cell adhesion molecule throughout the central nervous system of the adult rat: an immunohistochemical study. Neuroscience 49 419-436.

Bonfanti L, Merighi A \& Theodosis DT 1996 Dorsal rhizotomy induces transient expression of the highly sialylated isoforms of the neural cell adhesion molecule in neurons and astrocytes of the adult spinal cord. Neuroscience 74 619-623.

Cavarretta I, Magnaghi V, Ferraboschi P, Martini L \& Melcangi RC 1999 Interactions between type 1 astrocytes and LHRH-secreting neurons (GTI-1 cells): modification of steroid metabolism and possible role of TGF $\beta 1$. Journal of Steroid Biochemistry and Molecular Biology 71 41-47.

Dhandapani KM, Mahesh VB \& Brann DW 2003 Astrocytes and brain function: implications for reproduction. Experimental Biology and Medicine (Review) 228 253-260.

Doherty P, Walsh FS, Moore S \& Walsh FS 1991 Morpho-regulatory activities of NCAM and N-cadherin can be accounted for by a G-protein dependent activation of L and N-type neuronal calcium channels. Cell 67 21-33.

Fernandez-Galaz MC, Munoz RM, Villanua MA \& García-Segura LM 1999 Diurnal oscillation in glial fibrillary acidic protein in a peri-suprachiasmatic area and its relationship to the luteinizing hormone surge in the female rat. Neuroendocrinology 70 368-376.

Fryer HJ \& Hockfield S 1996 The role of polysialic acid and other carbohydrate polymers in neural structural plasticity. Current Opinion in Neurobiology 6 113-118.

Galbiati M, Magnaghi V, Martini L \& Melcanghi RC 2001 Hypothalamic TGF $\beta 1$ and bFGF mRNA expression is modified during the rat estrous cycle. Journal of Neuroendocrinology 13 483-489.

Galbiati M, Magnaghi V, Martini L \& Melcanghi RC 2002 Oestrogen via transforming growth factor alpha modulates basic fibroblast growth factor synthesis in hypothalamic astrocytes: in vitro observation. Journal of Neuroendocrinology 14 829-835.

Galbiati M, Saredi S \& Melcanghi RC 2003 Steroid hormones and growth factors act in an integrated manner at the levels of hypothalamic astrocytes: a role in the neuroendocrine control of reproduction. Annals of the New York Academy of Sciences 1007 162-168.

Garcia-Segura LM, Suárez I, Segovia S, Tranque PA, Calen JM, Aguilera P, Olmos G \& Guillamon A 1988 The distribution of glial fibrillary acidic protein in adult brain is influenced by neonatal levels of sex steroids. Brain Research 456 357-363.

Hoyk Z, Parducz A \& Theodosis DT 2001 The highly sialylated isoforms of the neural cell adhesion molecule is required for estradiol induced morphological synaptic plasticity in the adult arcuate nucleus. European Journal of Neuroscience 13 649-656.

Jansen HT, Cutter C, Hardy S, Lehman MN \& Goodman RL 2003 Seasonal plasticity within the GnRH system of the ewe: changes in identified GnRH inputs and glial association. Endocrinology 144 3663-3676. 
Kaur G, Heera PK \& Srivastava LK 2002 Neuroendocrine plasticity in $\mathrm{GnRH}$ release during rat estrous cycle: correlation with molecular markers of synaptic remodeling. Brain Reasearch 95 421-431.

King JC, Ronsheim PM \& Rubin BS 1995 Dynamic relationships between LHRH neuronal terminals and the end-feet of tanycytes in cycling rats revealed by confocal microscopy. Proceedings of the 25th Annual Meeting of the Society for Neuroscience. Abstract 21265.

Kohama SG, Goss JR, McNeill TH \& Finch CE 1995 Glial fibrillary acidic protein mRNA increases at proestrus in the arcuate nucleus of mice. Neuroscience Letters 183 164-166.

Levine JE 1997 New concepts of the neuroendocrine regulation of gonadotropin surge in rats. Biology of Reproduction 56 293-302.

Ma YJ, Hill DF, Junier MP, Costa ME, Felder SE \& Ojeda SR 1994 Expression of epidermal growth factor receptor changes in the hypothalamus during the onset of female puberty. Molecular and Cellular Neuroscience 5 246-262.

Melcangi RC, Cavaretta I, Magnaghi V, Martini L \& Galbiati M 2001 Interaction between growth factors and steroids in the control of LHRH secreting neurons (Review). Brain Research 37 223-234.

Melcangi RC, Martini L \& Galbiati M 2002 Growth factors and steroid hormones: a complex interplay in the hypothalamic control of reproductive functions. Progress in Neurobiology 67 421-449.

Naftolin F, Mor G, Horvath TL, Luquin S, Fajer AB, Kohen F \& García-Segura LM 1996 Synaptic remodeling in the arcuate nucleus during the estrous cycle is induced by estrogen and precedes the preovulatory gonadotropin surge. Endocrinology 137 5576-5580.

Ojeda SR, Prevot V, Heger S, Lomniczi A, Dziedzic B \& Alison M 2003 Glia-to-neuron signaling and the neuroendocrine control of female puberty (Review). Annals of Medicine 35 244-255.

Olsen M, Zuber C, Roth J, Linnemann D \& Bock E 1995 The ability to re-express polysialylated NCAM in soleus muscle after denervation is reduced in aged rats compared to young adult rats. International Journal of Developmental Neuroscience 13 97-104.

Prevot V, Croix D, Bouret S, Dutoit S, Tramu G, Stefano GB \& Beauvillain JC 1999 Definitive evidence for the existence of morphological plasticity in the external zone of the median eminence during the rat estrous cycle: implication of neuro-glio endothelial interactions in gonadotropin-releasing hormone release. Neuroscience 94 809-819.

Prevot E, Bouret S, Croix D, Alonso G, Jennes L, Mitchell V, Routtenberg A \& Beauvillain JC 2000 Growth associated protein-43 messenger ribonucleic acid expression in gonadotropin-releasing hormone neurons during the rat estrous cycle. Endocrinology 141 1648-1657.

Rao IM \& Mahesh VB 1986 Role of progesterone in modulation of the preovulatory surge of gonadotropins and ovulation in the
PMSG-primed immature rat and the adult rat. Biology of Reproduction 35 1154-1161.

Rutishauser U \& Landmesser L 1996 Polysialic acid in the vertebrate nervous system: a promoter of plasticity in cell-cell interactions. Trends in Neuroscience 19 422-427.

Sarkar DK Chiappa SA Fink G \& Sherwood NM 1976 GnRH surge in proestrous rats. Nature 26 4461-4463.

Seki T \& Arai Y 1991 Expression of highly polysialylated NCAM in the neocortex and piriform cortex of the developing and the adult rat. Anatomy of Embryology 184 395-401.

Seki T \& Arai Y 1993 Highly polysialylated neural cell adhesion molecule (NCAM-H) is expressed by newly generated granule cells in the dentate gyrus of the adult rat. Journal of Neuroscience $\mathbf{1 3}$ 2351-2358.

Silverman RC, Gibson MJ \& Silverman AJ 1991 Relationship of glia to GnRH axonal outgrowth from third ventricular grafts in hpg hosts. Experimental Neurology 114 259-274.

Stone DJ, Song Y, Anderson P, Krohn KK, Finch CE \& Rozovsky I 1998 Bidirectional transcription regulation of glial fibrillary acidic protein by estradiol in vivo and in vitro. Endocrinology 139 3202-3209.

Theodosis DT, Rougon G \& Poulain D 1991 Retention of embryonic features by an adult neuronal system capable of plasticity: polysialylated neural cell adhesion molecule in the hypothalamoneurohypophysial system. PNAS 88 5494-5498.

Theodosis DT, Bonhomme R, Vitiello S, Rougon G \& Poulain DA 1999 Cell surface expression of polysialic acid on NCAM is a prerequisite for activity-dependent morphological neuronal and glial plasticity. Journal of Neuroscience 19 10228-10236.

Theodosis DT, Piet R, Poulain DA \& Oliet SHR 2004 Neuronal, glial and synaptic remodeling in the adult hypothalamus: functional consequences and role of cell surface and extracellular matrix adhesion molecules. Neurochemistry International 45 491-501.

Viguie C, Jansen HT, Glass JD, Watanabe M, Billings HJ, Coolen L, Lehman MN \& Karsch FJ 2001 Potential for polysialylated form of neural cell adhesion molecule-mediated neuroplasticity within the gonadotropin-releasing hormone neurosecretory system of the ewe. Endocrinology 142 1317-1324.

Yoshida K, Rutishauser U, Crandall JE \& Schwarting GA 1999 Polysialic acid facilitates migration of luteinizing hormone-releasing hormone neurons on vomeronasal axons. Journal of Neuroscience 19 794-801.

Received 4 May 2005

Accepted 27 May 2005 